This item was submitted to Loughborough's Research Repository by the author.

Items in Figshare are protected by copyright, with all rights reserved, unless otherwise indicated.

\title{
SPH-FEM simulation of shaped-charge jet penetration into double hull: a comparison study for steel and SPS
}

PLEASE CITE THE PUBLISHED VERSION

http://dx.doi.org/10.1016/j.compstruct.2016.08.002

PUBLISHER

(C) Elsevier Ltd.

VERSION

AM (Accepted Manuscript)

\section{PUBLISHER STATEMENT}

This work is made available according to the conditions of the Creative Commons Attribution-NonCommercialNoDerivatives 4.0 International (CC BY-NC-ND 4.0) licence. Full details of this licence are available at: https://creativecommons.org/licenses/by-nc-nd/4.0/

\section{LICENCE}

CC BY-NC-ND 4.0

\section{REPOSITORY RECORD}

Zhang, Zhifan, Longkan Wang, Vadim V. Silberschmidt, and Shiping Wang. 2019. "SPH-FEM Simulation of Shaped-charge Jet Penetration into Double Hull: A Comparison Study for Steel and SPS". figshare. https://hdl.handle.net/2134/23022. 


\title{
SPH-FEM simulation of shaped-charge jet penetration into double hull: A comparison study for steel and SPS
}

\author{
Zhifan Zhang $^{\mathrm{a}, \mathrm{b}}$, Longkan Wang ${ }^{\mathrm{a}}$, Vadim V. Silberschmidt ${ }^{\mathrm{b}}$, Shiping Wang ${ }^{\mathrm{a}, *}$ \\ ${ }^{a}$ College of Shipbuilding Engineering, Harbin Engineering University, Harbin 150001, China \\ ${ }^{\mathrm{b}}$ Wolfson School of Mechanical, Electrical and Manufacturing Engineering, Loughborough University, Leicestershire LE11 $3 T U$, UK
}

Keywords:

Shaped-charge jet

$\mathrm{SPH}$

FEM

SPS

Damage response

\begin{abstract}
A B S T R A C T
A high-speed metal jet capable to cause severe damage to a double-hull structure can be produced after detonation of a shaped charge. A Smoothed Particle Hydrodynamics (SPH) method with a mesh-free and Lagrange formulations has natural advantages in solving extremely dynamic problems. Hence, it was used to simulate the formation process of a shaped-charge jet. A Finite Element Method (FEM) is suitable for a structural analysis and is highly efficient for simulations of a complex impact process in a relatively short time; therefore, it was applied to develop a double-hull model. In this paper, a hybrid algorithm fully utilizing advantages of both SPH and FEM is proposed to simulate a metal-jet penetration into a double hull made of different materials - steel and SPS (Sandwich Plate System). First, a SPH-FEM model of a sphere impacting a plate was developed, and its results were compared with experimental data to validate the suggested algorithm. Second, numerical models of steel/SPS double-hull subjected to a shapedcharge jet were developed and their results for jet formation, a penetration process and a damage response were analysed and compared. The obtained results show that the velocity of the metal jet tended to decrease from its tip to the tail during its formation process. The jet broke into separate fragments after the first steel shell was penetrated, causing the damage zone of the second shell that grew as a result of continuous impact by fragments. As for the SPS structure, its damage zone was smaller, and the jet trended to bend becoming thinner due to the resistance of the composite layer. It was found that the polyurethane layer could have a protective effect for the second shell.
\end{abstract}

\section{Introduction}

Shaped charges have received considerable international attention in recent years and are effectively used in military and civilian fields [1-12]. Molinari [1] used a finite-element Lagrangian code to simulate a process from jet formation to its penetration of a plate. Hazell et al. [2] numerically and experimentally investigated penetration of shaped charge jets into explosively driven brittle materials. Wu et al. [3] adopted an Arbitrary Lagrangian-Eulerian (ALE) method to study the penetration property of an explosive formed projectile (EFP) into an armour plate. However, computational difficulties can be caused by element distortion when traditional mesh-based methods are used to deal with nonlinear problems of high-speed impact. A meshless smoothed particle hydrodynamics (SPH) method [4-10] can overcome limitations related to the distorted mesh caused by large deformation. Feng et al. [7] applied

\footnotetext{
* Corresponding author.

E-mail address: wangshiping@hrbeu.edu.cn (S. Wang).
}

the SPH method to study explosion-driven metal deformation and jet formation as well as penetrating effects. Liu et al. [8] established a SPH model of a shaped charge with a hollow cavity and analysed pressure and density distributions in a detonation process. BaêtaNeves and Ferreira [9] successfully applied a two-dimensional SPH formulation using cylindrical coordinates to simulate a jetformation process for a cylindrical-shaped charge (CSC). All these studies indicate that the SPH method [7-12] has natural advantages in simulating the process of a metal-jet formation. Hence, it is utilized here to develop a model of a shaped charge.

A metal jet with high speed and high energy can cause severe damage to a structure. Hence, special protection designs attracted a wide attention in the recent years. With the development of materials science and technology, traditional materials are gradually substituted with advanced ones [13-17] in engineering in order to improve impact resistance of structures. Although steels have been widely used as armour materials in defence applications, they have a disadvantage of a relatively high density. Hence, sandwich plate systems (SPSs) [18-22] with lower density and numerous other advantages, such as enhanced blast and ballistic 
resistance, has been extensively employed for aerospace and marine applications. Although SPH approaches can be applied to problems with severe distortions, they are generally not as good as the finite-element method (FEM) [23-27] - a mature scheme with a high efficiency, suitable for structural applications. Therefore, alongside the SPH approach, the FEM is applied here to model steel/SPS structures and analyse their damage response, considerable effort was dedicated to coupled SPH-FEM methods [28-38] by various researchers in the past years. Johnson et al. [28,29] developed a master-slave method to describe a contact between SPH particles and finite elements and used it to simulate highvelocity impacts. Zhang et al. [32,33] effectively used a SPH-FEM coupling algorithm to treat the problem of a cylindrical projectile impacting a soft target. However, few studies were published on processes of a shaped-charge jet with high-speed impacting of SPS structures and their comparison with responses of steel shells.

In this paper, SPH and FEM are combined to fully utilize their advantages in simulations of the entire process of jet formation and target penetration. First, a simulation of a high-speed sphere (modelled with SPH particles) impacting a plate (simulated with finite elements) is performed, and its results are compared with experimental data to verify the validity and feasibility of the presented SPH-FEM method. Second, SPH-FEM models of shapedcharge jets penetrating double-hull structures are developed for two different materials - steel and SPS - were discussed. Finally, results for jet formation, a penetration process and a damage response are analysed.

\section{SPH-FEM models}

\subsection{Theory}

\subsubsection{SPH method}

A Lagrangian formulation of the motion equations is used in the SPH method [39-51]; it is meshfree and, therefore, very efficient for simulation of high-velocity impacts. Hence, a shaped-charge model was built with the SPH method in order to investigate the process of formation of a metal jet and its penetration performance. Two key steps are included in the formulation of SPH approximations. In the first step - kernel approximation - a field function $f(x)$ is approximated in a continuous form, which can be expressed as [51]

$\langle f(x)\rangle=\int_{\Omega} f\left(x^{\prime}\right) W\left(x-x^{\prime}, h\right) d x^{\prime}$,

where $\langle f(x)\rangle$ is the approximated value; $W$ is the smoothing function representing a weighted contribution; $h$ is the smooth length; $x$ is the position vector of the particle.

In the second step - particle approximation - the field function $f(x)$ and its derivative $\nabla f(x)$ are approximated as weighted sum over surrounding particles within the support domain, which can be written as $[51]$

$$
\begin{aligned}
& \left\langle f\left(\boldsymbol{x}_{i}\right)\right\rangle=\sum_{j}^{N} \frac{m_{j}}{\rho_{j}} f\left(\boldsymbol{x}_{j}\right) W_{i j}, \\
& \left\langle\nabla \cdot f\left(\boldsymbol{x}_{i}\right)\right\rangle=\sum_{j=1}^{N} \frac{m_{j}}{\rho_{j}} f\left(\boldsymbol{x}_{j}\right) \cdot \nabla_{i} W_{i j},
\end{aligned}
$$

where $\left\langle f\left(\boldsymbol{x}_{i}\right)\right\rangle$ is the approximated value for particle $i$; $N$ is the number of particles in the support domain; $\boldsymbol{x}, m$ and $\rho$ denote the coordinates, mass and density, respectively; $W_{i j}$ is the smoothed function of a pair of particles $i$ and $j$ (the cubic spline function is employed in this paper).
Navier-Stokes equations are applied for hydrodynamics of fluids and solids with material strength. A detailed derivation of a SPH particle approximation for the continuity, momentum and energy equations can be found in [51]:

$$
\begin{aligned}
& \frac{d \rho_{i}}{d t}=\rho_{i} \sum_{j=1}^{N} m_{j} \cdot v_{i j}^{b} \frac{\partial W_{i j}}{\partial x_{i}^{b}}, \\
& \left.\frac{d \boldsymbol{v}_{i}^{a}}{d t}=-\sum_{j=1}^{N} \frac{m_{j}}{\rho_{j}} \frac{\sigma_{i}^{a b}+\sigma_{j}^{a b}}{\rho_{i}}+\Pi_{i j}\right) \frac{\partial W_{i j}}{\partial x_{i}^{b}}, \\
& \left.\frac{d e_{i}}{d t}=\frac{1}{2} \sum_{j=1}^{N} \frac{m_{j}}{\rho_{j}} \frac{\sigma_{i}^{a b}+\sigma_{j}^{a b}}{\rho_{i}}+\Pi_{i j}\right) \boldsymbol{v}_{i j}^{b} \frac{\partial W_{i j}}{\partial x_{i}^{b}},
\end{aligned}
$$

where $\boldsymbol{v}, e$ and $t$ denote the velocity, energy and time, respectively; $a$ and $b$ indicate the directions along the axes; $\Pi_{i j}$ is artificial viscosity [51]; $\sigma$ is stress, composed of isotropic pressure and shear stress.

Various schemes are used to describe different components of a multi-material system.

(1) For the exploding gas, viscous shear stress can be neglected since its isotropic pressure $P$ is much larger. Here, since the exploding gas with high temperature and high pressure is generated after explosion initiation, this pressure can be presented with the Jones-Wilkins-Lee (JWL) equation of state (EoS), introduced in the following form [52]:

$$
P=A\left(1-\frac{\omega \eta}{R_{1}}\right) \exp \left(-\frac{R_{1}}{\eta}\right)+B\left(1-\frac{\omega \eta}{R_{2}}\right) \exp \left(-\frac{R_{2}}{\eta}\right)+\omega \eta \rho_{0} e
$$

where $\rho_{0}$ is the initial density; $A, B, R_{1}, R_{2}$ and $\omega$ are the fitting coefficients obtained from experiments; $e$ is detonation energy per unit mass; $\eta$ is the ratio of the density of detonation products to the initial density of the original explosive. The detailed parameters are listed in Table 1.

(2) For solids with high shear strength, stress can be presented as a combination of isotropic pressure $P$ and deviatoric stress tensor $S$. The isotropic pressure $P$ was computed with the Mie - Grüneisen EoS for solid media [53], written as

$P=\left(1-\frac{1}{2} \Gamma \eta\right) \cdot\left(\alpha \eta+\beta \eta^{2}+\gamma \eta^{3}\right)+\Gamma \rho e$,

where $\Gamma, \rho$ and $e$ denote the Grüneisen parameter, density and initial energy, respectively. In the case when $\eta>0$, $\alpha=\rho_{0} C_{s}^{2}, \quad \beta=\alpha\left[1+2\left(S_{s}-1\right)\right]$ and $\gamma=\alpha\left[2\left(S_{s}-1\right)+3\left(S_{s}-\right.\right.$ $\left.1)^{2}\right]$, where $C_{s}$ and $S_{s}$ are the linear participation coefficient of the impact velocity and particles velocity, and slope, respectively. Otherwise, a solid is in an expansion state, and then $\beta=\gamma=0$. The detailed parameters are listed in Table 2 .

Table 1

Parameters in Jones-Wilkins-Lee EoS for explosive gas [52].

\begin{tabular}{lllllll}
\hline$\rho_{0}\left(\mathrm{~kg} / \mathrm{m}^{3}\right)$ & $A(\mathrm{~Pa})$ & $B(\mathrm{~Pa})$ & $R_{1}$ & $R_{2}$ & $\omega$ & $e(\mathrm{~J} / \mathrm{kg})$ \\
\hline 1630 & $3.74 \times 10^{11}$ & $3.75 \times 10^{9}$ & 4.15 & 0.90 & 0.35 & $6.0 \times 10^{6}$ \\
\hline
\end{tabular}

Table 2

Parameters in Mie-Grüneisen EoS for copper [53].

\begin{tabular}{llllll}
\hline Material & $\rho_{0}\left(\mathrm{~kg} / \mathrm{m}^{3}\right)$ & $\Gamma$ & $C_{s}(\mathrm{~m} / \mathrm{s})$ & $S_{s}$ & $e(\mathrm{~J} / \mathrm{kg})$ \\
\hline Copper & 8960 & 2.0 & 3940 & 1.489 & 0 \\
\hline
\end{tabular}


The deviatoric stress tensor $S$ can be calculated from the stress rate $\dot{S}$ by integration, with $\dot{S}$ obtained from the Jaumann stress rate [51]. The Johnson-Cook model [54] and a von Mises yield criterion were applied to calculate yield strength and determine whether the stress should be renewed. If von Mises stress $\sigma_{\text {Mises }}$ was larger than the yield strength $Y$, the deviatoric stress $S$ should be modified. The Johnson-Cook model [54] defining the effects of stress rate and temperature is given as

$Y=\left[\sigma_{0}+B_{0} \varepsilon_{e}^{n}\right]\left[1+C \ln \left(\dot{\varepsilon}_{e} / \dot{\varepsilon}_{0}\right)\right]\left[1-\left(T^{*}\right)^{M}\right]$

where $\sigma_{0}, C$ and $M$ denote the static yield strength, strain-ratestrengthening and thermal-softening coefficients, respectively; $B_{0}$ and $n$ are the strain-hardening parameters; $\varepsilon_{e}$ is the equivalent plastic strain, i.e. $\varepsilon_{e}=2 \sqrt{3 I_{2}} / 3\left(I_{2}\right.$ is the second invariant of the deviatoric stress tensor); $\dot{\varepsilon}_{e}$ and $\dot{\varepsilon}_{0}$ are rates of the equivalent plastic strain and reference strain, respectively; $T^{*}$ is dimensionless temperature corresponding to the current level $T$, i.e. $T^{*}=$ $\left(T-T_{r}\right) /\left(T_{m}-T_{r}\right)$, where $T_{r}$ is room temperature, $T_{m}$ is the melting point and $T=T_{r}+\left(e-e_{0}\right) /\left(M C_{v}\right)$, where $e, e_{0}$ and $C_{v}$ denote specific internal energy, initial specific internal energy and specific heat. The detailed parameters are listed in Table 3.

\subsubsection{FEM}

The FEM has well-known advantages to simulate accurately and highly efficiently a complex impact process in a relatively short time. Here, the focus is on SPS structures with a reduced weight and increased fatigue resistance widely used in specific applications. Therefore, two models of double-hull systems with different materials - steel and SPS - were developed with the FEM. In order to investigate their impact resistance, a comparison study of jet formation, penetration and damage response was conducted for these two cases. For the SPS structure, two steel shells were bonded to a compact polyurethane core, forming a steelpolyurethane-steel sandwich structure. Masses of the structures in both cases were almost equal. A Cowper-Symonds strain rate model [55-57] was used for the steel and the polyurethane, expressed as

$\sigma_{y}=\left[1+\left(\frac{\dot{\varepsilon}}{C}\right)^{\frac{1}{p}}\right]\left(\sigma_{0}+\beta E_{p} \varepsilon_{p}^{e f f}\right)$,

where $\sigma_{0}$ is the initial yield stress; $\dot{\varepsilon}$ is the strain rate; $C$ and $p$ are the Cowper-Symonds coefficients; $\beta$ is the stiffness parameter; $\varepsilon_{p}^{e f f}$ is the effective plastic strain; $E_{p}$ is the plastic hardening modulus: $E_{p}=\frac{E E_{\tan }}{E-E_{\tan }}$, where $E$ and $E_{\tan }$ are the elasticity and tangent moduli. The magnitudes of parameters used in numerical simulations are listed in Table 4.

\subsubsection{A hybrid SPH-FEM algorithm}

As discussed, the SPH method has advantages in simulating processes of initiation of a shaped charge and jet formation, since, due to its mesh less and Lagrangian nature, it is applicable to problems of large deformation and can be used easily to capture material interfaces and free surfaces. The FEM is a conventional numerical method used to predict the performance of structures and suitable to study the sandwich material. Hence, a hybrid algorithm was utilized to simulate the entire process of a jet formation and its penetration into a SPS/steel double-hull structure. The shaped charge
Table 4

Parameters in Cowper-Symonds model for steel and polyurethane [55-57].

\begin{tabular}{|c|c|c|c|c|c|c|c|c|}
\hline Materials & $\begin{array}{l}\rho_{0} \\
\left(\mathrm{~kg} / \mathrm{m}^{3}\right)\end{array}$ & $v$ & $\begin{array}{l}E \\
(\mathrm{GPa})\end{array}$ & $\begin{array}{l}E_{\tan } \\
(\mathrm{MPa})\end{array}$ & $\begin{array}{l}\sigma_{y} \\
(\mathrm{MPa})\end{array}$ & $\beta$ & $P$ & C \\
\hline Steel & 7850 & 0.3 & 206 & 763 & 315 & 0 & 5 & 40.4 \\
\hline Polyurethane & 1100 & 0.36 & 0.75 & - & 18 & 0 & - & - \\
\hline
\end{tabular}

and the double-hull structure were modelled with the SPH method and FEM, respectively. A penalty method was used to treat the interaction between metal-jet particles and target elements during the entire process of jet penetration into the structure. A detailed diagram of the hybrid algorithm is shown in Fig. 1, with its upper and lower parts presenting computation processes of SPH and FEM $[29,31,58]$. At the interface of the SPH and FEM domains, when a particle contacted the surface, a contact force was generated and parameters of particles and nodes were renewed. Otherwise, these methods worked just as two independent processes. A node-tosurface contact algorithm was applied in simulations, with the jet particles and the target used as the slave and master sets, respectively.

\subsection{Model verification}

To verify the developed approach, an initial study was implemented focused on experimental evidence. A model of an impact between an aluminium sphere and a copper plate is presented in Fig. 2. The magnitudes of radius $R_{s}$ and velocity $v$ of the sphere were respectively $5 \mathrm{~mm}$ and $5750 \mathrm{~m} / \mathrm{s}$. The plate made of copper had a thickness $t_{p}=1.5 \mathrm{~mm}$; the material parameters were found in [59]. 15,666 particles were used to model an SPH-domain of the aluminium sphere and 21,000 nodes for a FEM-domain of the copper plate.

A comparison of the obtained results for a shape of a debris cloud and a crater diameter is presented in Fig. 3 and Table 5. A circular hole was caused by the impact of the sphere, with a debris cloud generating (Fig. 3). It was obvious that the numerical results

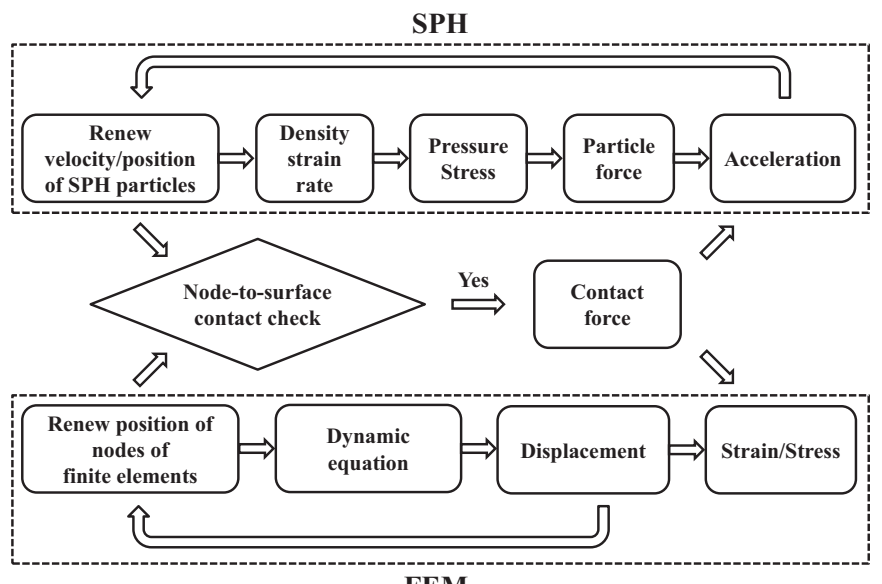

FEM

Fig. 1. Flowchart of hybrid SPH-FEM method.

Table 3

Parameters in Johnson-Cook constitutive model for copper [54].

\begin{tabular}{|c|c|c|c|c|c|c|c|c|c|}
\hline Material & $\sigma_{0}(\mathrm{MPa})$ & $B_{0}(\mathrm{MPa})$ & $\dot{\varepsilon}_{0}\left(\mathrm{~s}^{-1}\right)$ & $n$ & $C$ & $M$ & $T_{m}(\mathrm{~K})$ & $T_{r}(\mathrm{~K})$ & $\left.C_{v} \mathrm{~J} / \mathrm{kg} \cdot \mathrm{K}\right)$ \\
\hline Copper & 90 & 292 & 1 & 0.31 & 0.025 & 1.09 & 1356 & 288 & 383 \\
\hline
\end{tabular}




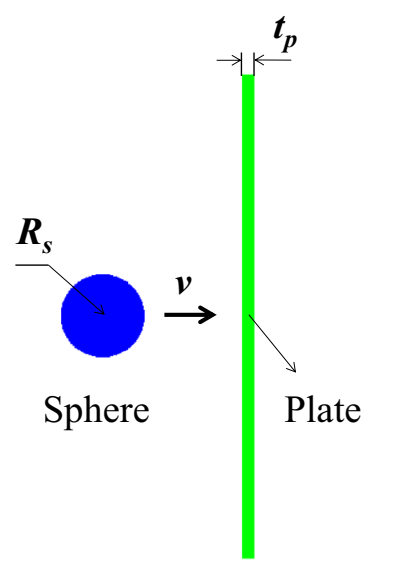

Fig. 2. A1-Cu impact model.

obtained with the new hybrid scheme for the shape of debris cloud were in good agreement with the experimental data.

Moreover, magnitudes of the crater diameter obtained from the numerical simulation and in the experiments were, respectively, $22.6 \mathrm{~mm}$ and $21.2 \mathrm{~mm}$ with an error less than $7 \%$. Also, a difference between the shape factors (ratios of length to width) of the debris clouds did not exceed $1 \%$ (Table 5 ). It could be concluded from this comparison the suggested numerical scheme reproduces the experimental results in a reasonable way, proving feasibility of the hybrid algorithm based on the combination of SPH and FEM.

\subsection{Numerical simulations}

The next step was to apply the suggested approach to a doublehull problem. A SPH model of a shaped charge is presented in Fig. 4. TNT was chosen as the explosive, with height $h=400 \mathrm{~mm}$ and radius $R=160 \mathrm{~mm}$; it was detonated from the centre at the top. Copper was used as the material of the hemispherical liner, with the magnitudes of outer radius $R=160 \mathrm{~mm}$ and thickness $d=8 \mathrm{~mm}$. The SPH models were discretized with non-uniform particle spacing, for the TNT $d x_{1}=4 \mathrm{~mm}$ and for the copper $d x_{2}=2 \mathrm{~mm}$. To model the SPH domains of the charge and the liner, 217,372 and 171,886 particles were used, respectively.

A FEM model of a double-hull structure is also shown in Fig. 4 with its dimensions. The distances $h_{1}$ between two shells and $h_{2}$ between the first shell and the charge bottom were respectively $300 \mathrm{~mm}$ and $500 \mathrm{~mm}$. For steel shells (Case 1), the magnitudes of thicknesses of two shells $d_{1}$ and $d_{2}$ were $10 \mathrm{~mm}$ and $20 \mathrm{~mm}$, respectively. 104,120 elements were used to model the FEM domain of each shell. In the SPS (Case 2), each shell had three lay-
Table 5

Comparison of results for crater diameter and shape of debris cloud.

\begin{tabular}{lll}
\hline Methods & Crater diameters $(\mathrm{mm})$ & Ratio of length to width \\
\hline Experimental & 21.2 & 1.39 \\
Simulation & 22.6 & 1.40 \\
\hline
\end{tabular}

ers, external ones made of steel and the internal one of polyurethane. Masses of the structures in both cases were almost equal. Cases and thicknesses of layers were listed in Table 6.

\section{Results and discussion}

\subsection{Formation of shaped-charge jet}

According to the numerical simulations, a metal jet was formed gradually under the effect of the detonation wave after the explosive was detonated. A velocity distribution for the liner at specific moments is shown in Fig. 5. It was evident from Fig. 5(a) that the liner was squeezed under the effect of the detonation wave. As a result of its axial tension and radial compression the high-speed (about $3147 \mathrm{~m} / \mathrm{s}$ ) metal jet formed at about $100 \mu \mathrm{s}$, with the original top of the liner rolling over to become the jet head. A stretching jet was produced due to the velocity gradient - the velocity decreased from tip to tail - as shown in Fig. 5(b), with the jethead velocity reaching the maximum (about $4150 \mathrm{~m} / \mathrm{s}$ ) at about $130 \mu \mathrm{s}$. As the jet elongated increasingly, the jet-head velocity diminished to about $3912 \mathrm{~m} / \mathrm{s}$ at $t \approx 180 \mu \mathrm{s}$, forming a "pestle" with a low speed (about $200 \mathrm{~m} / \mathrm{s}$ ) in the tail of the jet in Fig. 5(c). It was found that a velocity distribution presented a decreasing trend from its tip to the tail, in good accordance with the laws of physics. After that, the jet began to impact the structure.

Together with the jet formation and its velocity distribution, the jet-head velocity is one of the most significant factors. Hence, results of comparison for this velocity for the studied cases are discussed in this section (see also Fig. 6). It was found that these two cases shared a similar trend - the velocity reduced faster after the penetration and eventually stayed steady. As the metal jet formed gradually, the jet-head velocity peaked at about $4150 \mathrm{~m} / \mathrm{s}$ at about $130 \mu \mathrm{s}$. After that, it decreased slowly under the effect of the velocity gradient. At about $180 \mu$ s, it declined abruptly after the jet impacted the first shell; this decline was more rapid in Case B, indicating that the SPS reduced the jet-head velocity effectively. The jet began to penetrate the second shell at about $315 \mu$ s with the velocity diminishing again. At a final stage, Case A had a slightly larger steady velocity than Case B. Consequently, it was found that the resistance effect of SPS on jet-head velocity was more obvious than that of steel.
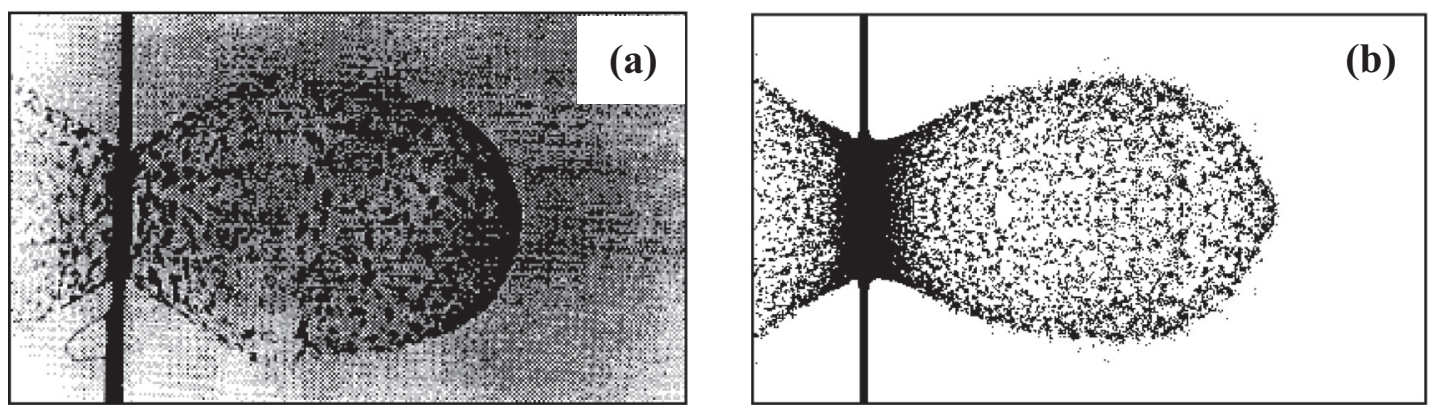

Fig. 3. Experimental [59] (a) and numerical (b) results for shape of debris cloud. 

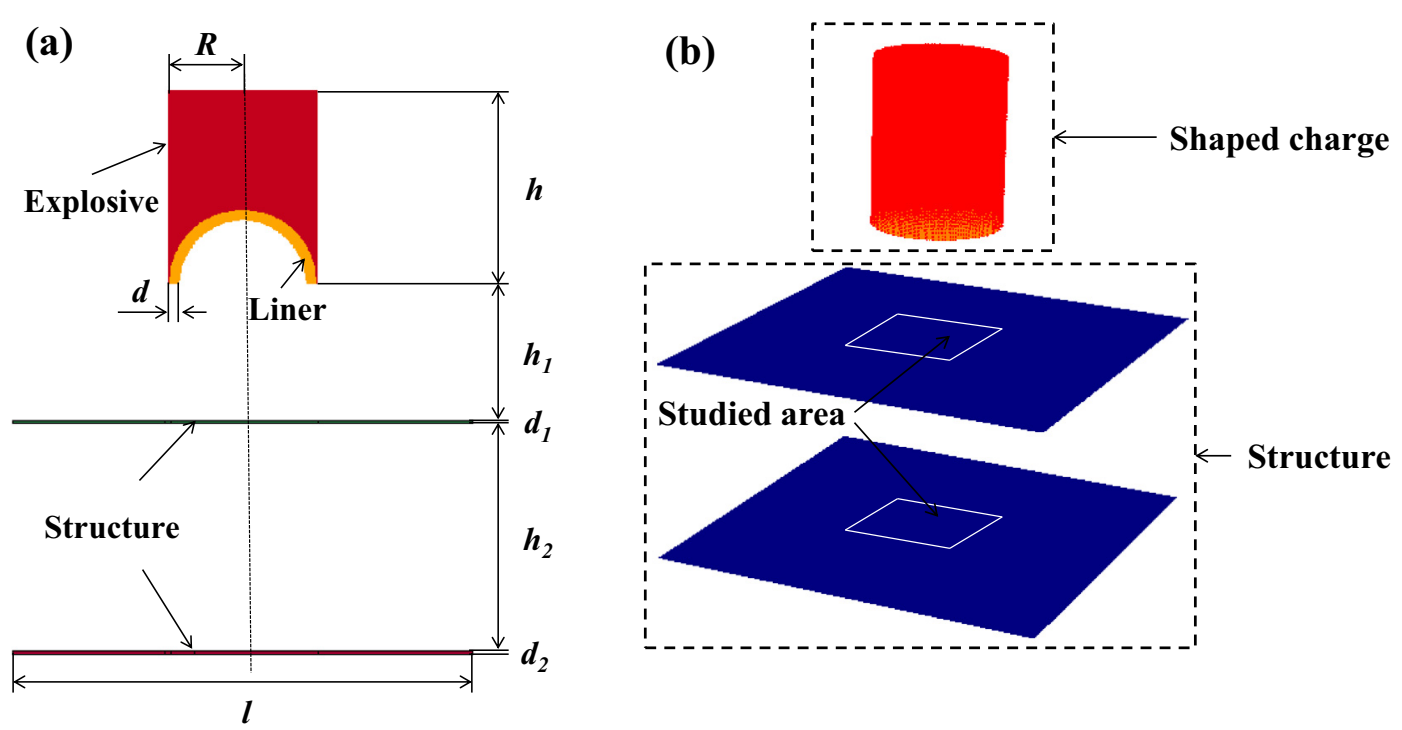

Fig. 4. Model of shaped charge damaging double-hull structure: (a) cross-section; (b) 3D model.

Table 6

Thickness of each layer in two cases (in $\mathrm{mm}$ ).

\begin{tabular}{llll}
\hline Shell No. & Case A & Case B & \\
\cline { 2 - 4 } & Steel & SPS & 4 \\
\hline 1 & 10 & First layer & 12 \\
& & Second layer & 4 \\
& & Third layer & 8 \\
& 20 & First layer & 24 \\
& & Second layer & 8 \\
\hline
\end{tabular}

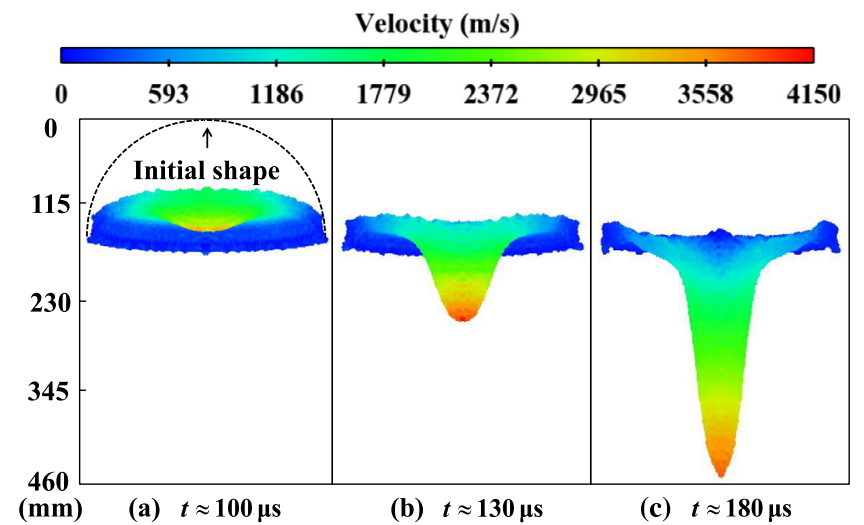

Fig. 5. Velocity distribution in liner in process of jet formation: (a) initial jet is formed; (b) jet-head velocity maximum reaches; (c) jet impacts structure.

\subsection{Penetration process}

An impact by the metal jet of the structure starts a process of jet penetration; Fig. 7 illustrates it. For Case A, the jet began to impact the first shell at about $180 \mu$ s (see Fig. 7(a)). Subsequently, the shell was penetrated, resulting in the jet breaking into separate fragments (shown in the rectangular region in Fig. 7(b)) at about $240 \mu \mathrm{s}$. After that, the jet elongated to several times of its initial length. At about $400 \mu \mathrm{s}$, the second shell was penetrated by several

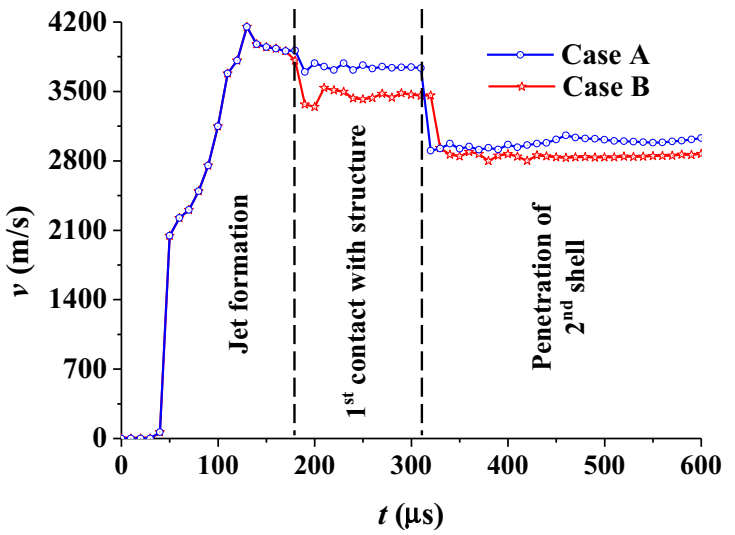

Fig. 6. Velocity-time curve.

fragments. A circular inset region in Fig. 7(c) shows that a part of the jet bounced back due to the accumulation of fragments. As for Case B, the process of the jet impacting two shells is shown in Fig. 7(d)-(f). The jet tended to bend as a result of a "squeezing" effect in Fig. 7(f). It was found from the comparison of the obtained results that the jet shape was similar when the first shell was impacted, yet different when it reached the second shell. Therefore, processes of the second-shell penetration are discussed in detail below.

Fig. 8 shows the process of the jet penetrating the second shell for Case A. The metal jet began to penetrate the second shell at about $315 \mu$ s (Fig. 8(a)). Before this, the jet broke into five parts - four fragments (denoted 1-4 in Fig. 8(a)) and the main part (5). At about $330 \mu \mathrm{s}$, the first fragment penetrated the shell, producing an initial hole (Fig. 8(b)). As the second and third fragments impacted the shell, the radius of the hole gradually increased (Fig. 8(c)-(d)). The fourth fragment reached the shell at about $455 \mu$ s and the main part of the jet began to penetrate the shell (Fig. 8(e)). As fragments accumulated, the partial jet bounced, resulting in the increase of the hole (Fig. 8(f)).

For Case B, the process of the jet penetrating the second shell is shown in Fig. 9. Under the effect of impact, the metal jet reached the shell causing a hole (Fig. 9(b)). At about $390 \mu$ s, the jet started 

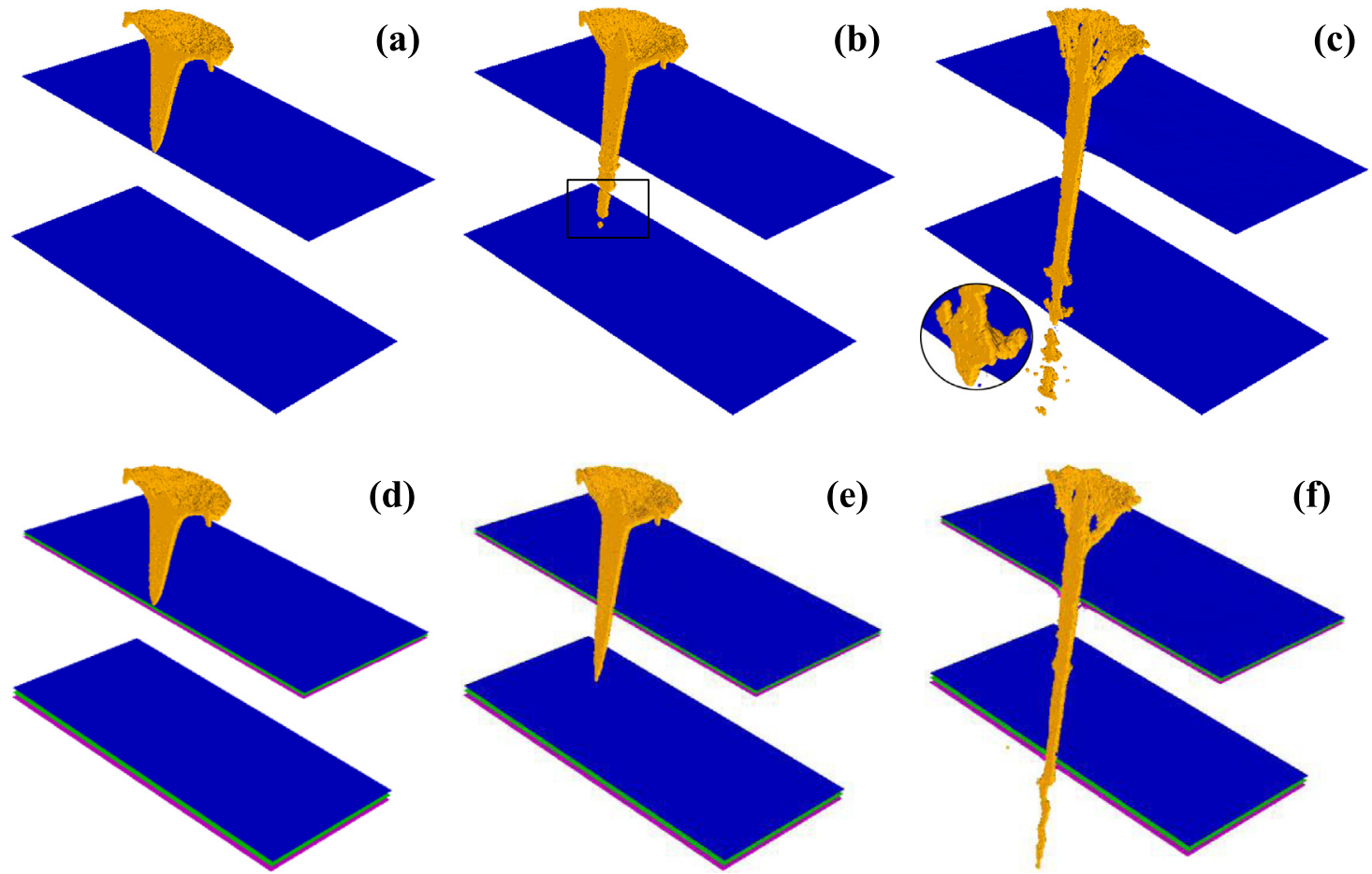

$t \approx 180 \mu \mathrm{S}$

$t \approx 240 \mu \mathrm{s}$

$$
t \approx 400 \mu \mathrm{s}
$$

Fig. 7. Process of jet penetration into structures at $180 \mu \mathrm{s}, 240 \mu$ s and $400 \mu \mathrm{s}$ : (a)-(c) Case A; (d)-(f) Cases B.
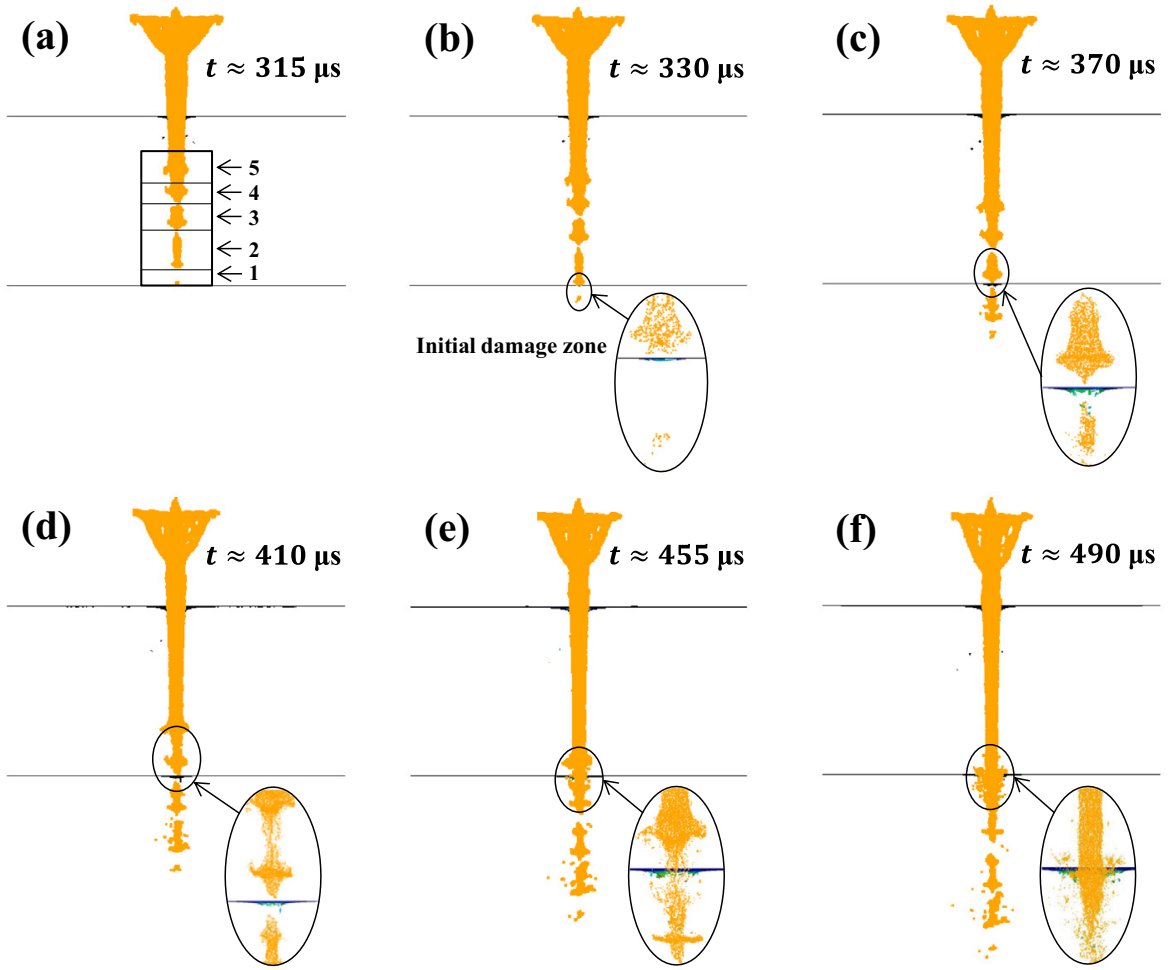

Fig. 8. Process of penetration of second shell in Case A. 

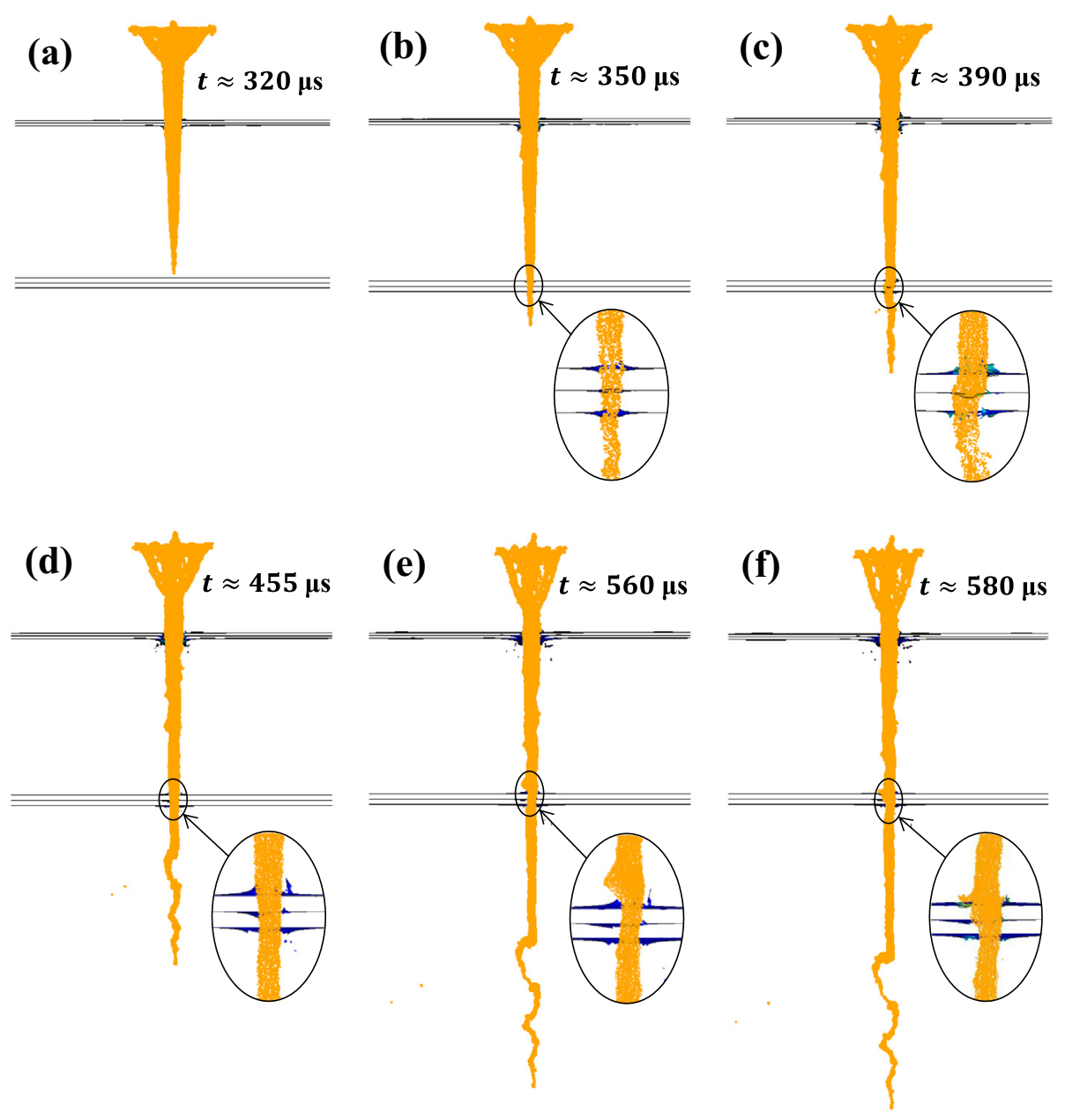

Fig. 9. Process of penetration of second shell in Case B.

bending due to the resistance effect of the SPS and the radius of the hole increased further as a result (Fig. 9(c)). It is obvious (Fig. 9(d)) that the hole in the second layer (polyurethane) was smaller than that in the first and third ones (steel). Besides, the jet narrowed in the SPS. At about $560 \mu \mathrm{s}$, a protruding part began to impact the shell. The jet's thinning could be clearly observed in Fig. 9(e). After that, the protruding part penetrated the shell causing a larger damage zone in the first layer at about $580 \mu \mathrm{s}$. It was found that the partial jet bounced in the opposite direction (Fig. 9(f)).

The comparison of results showed that the second shell in Case A was continuously penetrated by several fragments, yet in Case B the shell was directly penetrated. The jet's rebound was caused respectively by fragments accumulation and the protruding part in Cases A and B that might cause a greater damage to the shell. Besides, the jet trended to bend and became thin due to the resistance effect of the composite layer in Case B.

\subsection{Damage response}

Damage characteristics obtained in simulations are discussed in this section after studying evaluation of the jet's shape during the penetration process. Distributions of damage zones of the first shell in two cases were presented in Fig. 10. For Case A, the first shell was deformed and became thinner during the phase of plug failure at about $195 \mu$ s (Fig. 10(a)). As the plastic deformation occurred, a circular damage zone was caused at about $285 \mu \mathrm{s}$ (Fig. 10(b)), with several fragments produced around the hole. Under the jet impact, the shell was completely penetrated, and the damage zone increased gradually. Its edges tended to roll at about $450 \mu \mathrm{s}$ (Fig. 10(c)). In Case B, three layers began to be impacted by the jet, with a plastic deformation caused (Fig. 10 (d)). Plastic strain of the second layer (polyurethane) was higher than that of two other ones (steel). At about $285 \mu$ s, the three layers were damaged and a circular hole was generated at the centre; the damage zone of the third layer was slightly larger. As the further impact effect of the jet continued, the damage zone grew gradually.

A further comparison of evolution of damage zones in the first shell is presented in Fig. 11, in terms of dimensionless size (normalized with the TNT diameter), denoted $D$. These zones increased rapidly as the shell was penetrated, growing slowly with time after that. At about $600 \mu \mathrm{s}$, the magnitudes of $D$ were $0.2,0.21,0.21$ and 0.20 for Case A and three layers of Case B, respectively. Combining this with the results for failure modes in Fig. 10, the damge effects when the metal jet penetrated the first shell were found to be almost the same. 

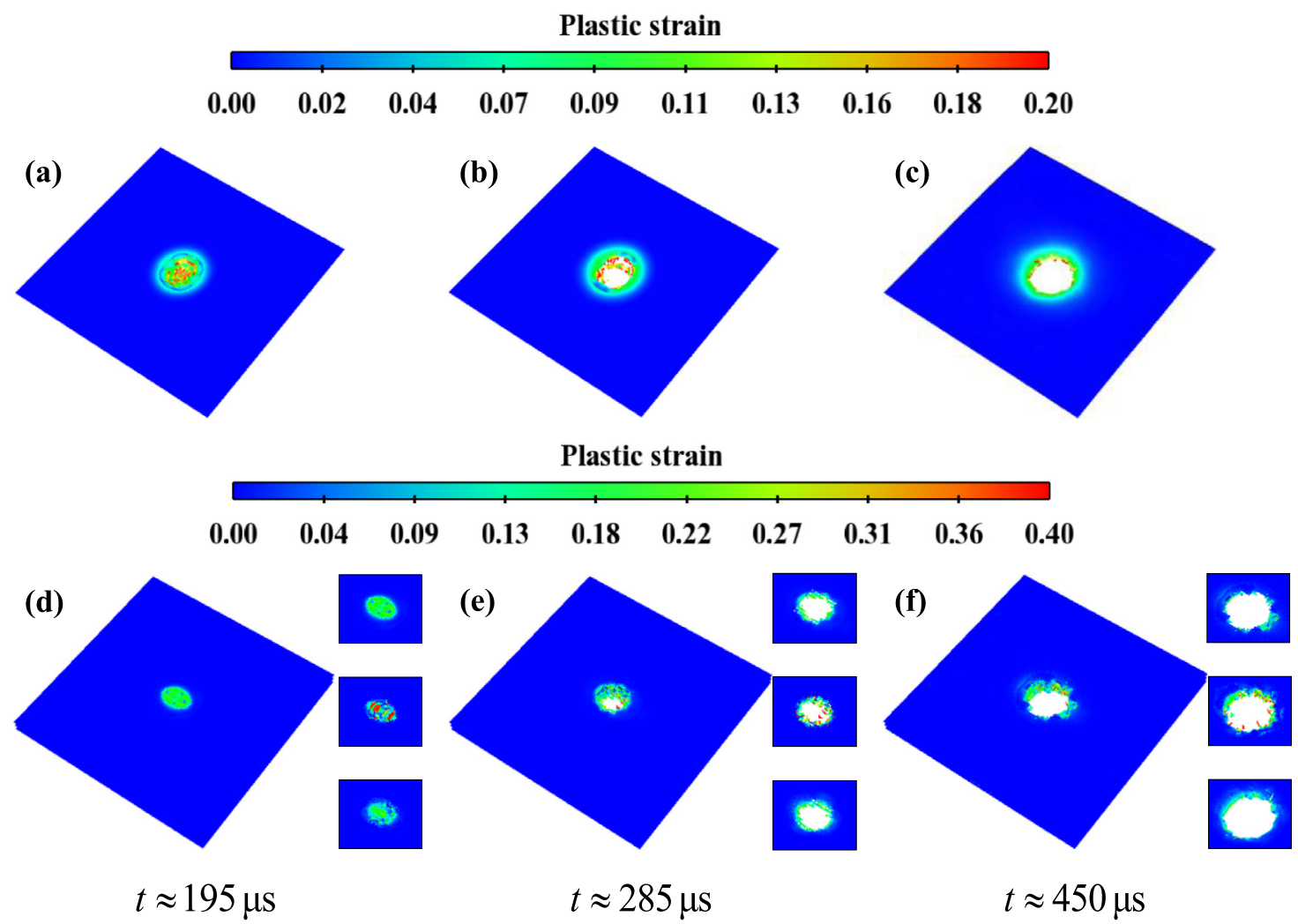

Fig. 10. Distribution of plastic strain in first shell at $t \approx 195 \mu \mathrm{s}, 285 \mu$ s and $450 \mu \mathrm{s}$ : (a)-(c) Case A; (d)-(f) Case B (insets show damage zones in three layers).

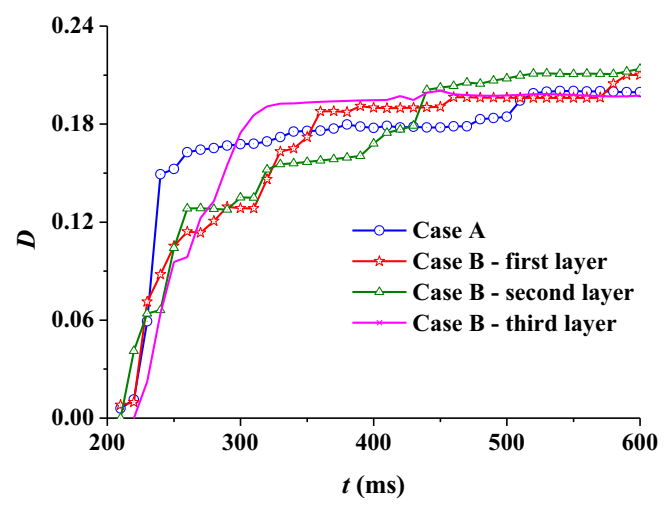

Fig. 11. Evolution of damage zone in first shell.

The evolution of damage zone in the second shell of the double hull is demonstrated in Fig. 12. For Case A, a spherical fragment 1 shown in Fig. 8 first impacted the second shell. As a result of the impact effect, the shell developed a pit with a greater plastic deformation, and shear failure occurred at the contact point. This fragment went through the shell at about $350 \mu$ s causing the initial damge zone, with a bulge sheared off (Fig. 12(a)). With further impact, the hole's radius increased gradually. The shape of the hole was circular at about $450 \mu$ s (Fig. 12(b)). After that, the main part of the jet began impacting the shell. The shape of the damage zone changed from circular to irregular, and the radius increased due to jet rebounding (Fig. 12(c)). In Case B, as a result of tensile tearing at the centre of the shell, the initial hole was produced (Fig. 12(d)). The jet at the interaction was compressed to become thinner as shown in Fig. 9(d) and (e). This phenomenon corresponds with results for the damage zones for the three layers, i.e. the radius of the second layer was smaller than that of the other ones. At about $600 \mu \mathrm{s}$, the holes transformed from a circular domain into an irregular one. The reason for this was probably that the jet became thin and was bent as the result of interaction between the jet and the structure.

After analysing the failure mode, evolution of the damage zone at the second shell was investigated as shown in Fig. 13. For Case $\mathrm{A}$, in the steep curve the kinks caused by continual penetration of fragments correspond to the moments in Fig. 8. In particular, the hole's radius surged in Fig. 8 at about $490 \mu$ s due to the accumulation of fragments and the jet's rebound, which could also be found in this curve. As for Case B, curves with fewer kinks trended to increase. A larger hole's radius for the first layer was caused by the protruding part and jet's rebound at about $580 \mu$ s. The magnitudes of $D$ were $0.24,0.19,0.11$ and 0.12 , respectively, for Case A and the three layers of Case B at about $600 \mu \mathrm{s}$. The hole's radius of the SPS was smaller than that of the steel shell. It indicated clearly that the second shell could be additionally protected by the polyurethane layer from damage caused by the metal jet.

\section{Conclusions}

In this paper, a hybrid SPH-FEM model was suggested to simulate the entire process of a metal-jet formation and its penetration into a double-hull structure. First, experimental results were compared with numerical ones to verify the effectiveness of the hybrid algorithm. Second, two cases with different materials - steel and SPS - were discussed, and comparison of the results obtained for formation of the shaped-charge jet, the penetration process and the damage response could be summarized as follows: 

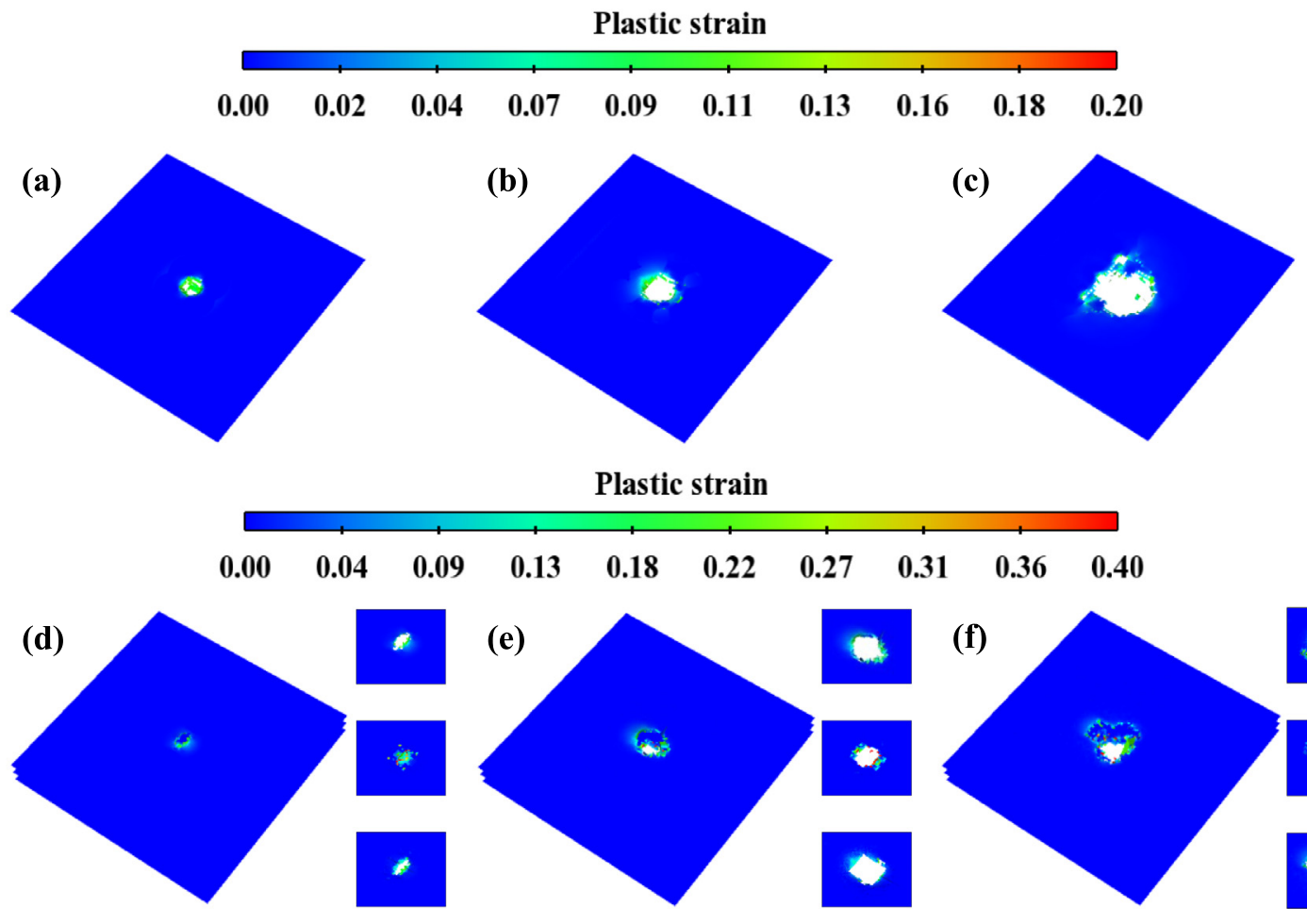

$0.13 \quad 0.18$

0.22

0.27

0.31

0.36

0.40

$$
t \approx 350 \mu \mathrm{s}
$$

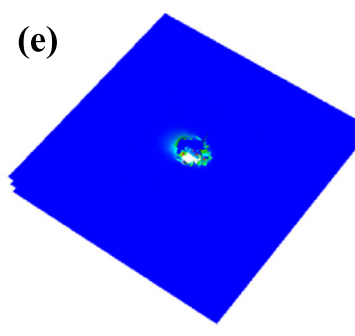

$t \approx 450 \mu \mathrm{s}$

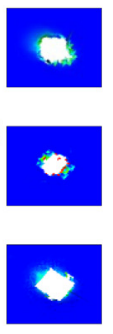

(f)

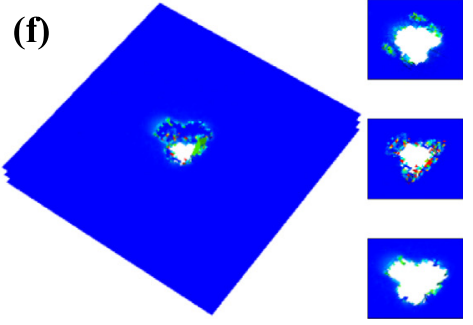

$$
t \approx 600 \mu \mathrm{s}
$$

Fig. 12. Distributions of plastic strain in second shell at $350 \mu \mathrm{s}, 450 \mu$ s and $600 \mu \mathrm{s}$ : (a)-(c) Case A; (d)-(f) Cases B (insets show damage zones in three layers).

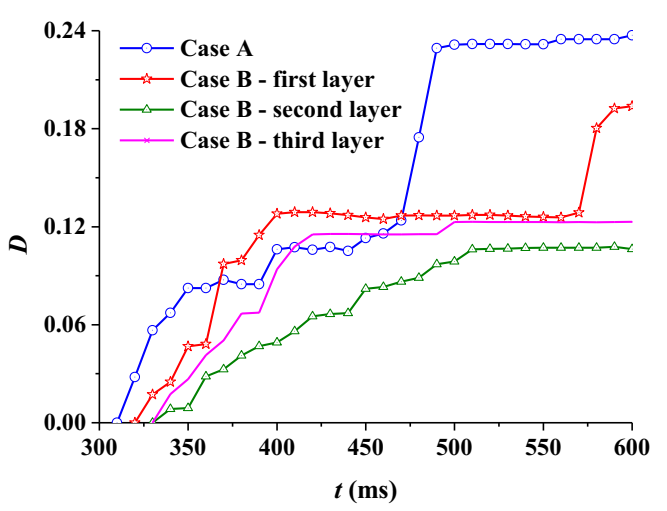

Fig. 13. Evolution of damage zone in second shell.

(1) The velocity of a metal jet with a linear distribution from tip to tail in the formation process tended to decrease, in good accordance with the laws of physics.

(2) The residual velocity in Case B was somewhat lower than that in Case A. It indicated that the resistance effect of SPS on the jet-head velocity was more pronounced than that of steel.

(3) During the penetration process, the metal jet broke into many fragments and, subsequently, they continuously impacted the second shell in Case A while the jet directly penetrated the SPS in Case B. Besides, due to the accumulation of fragments, the partial jet bounced and resulted in the increased size of the hole in Case A. The jet tended to bend, becoming thin due to the resistance effect of the composite layer in Case B.
(4) For the first shell of the double hull, the failure mode and the diameter of the hole in the two studied cases were similar. As for the second shell, the steel shell in Case A was initially damaged by a spherical fragment, with the bulge sheared off, and the damage zone increased gradually as it was penetrated by other fragments. In Case B, the initial damage was caused by tensile tearing. Besides, the damage zone was smaller than that in Case A, indicating that an additional protective effect for the second shell is provided by the polyurethane layer.

\section{Acknowledgement}

This work is supported by the National Natural Science Foundation of China (U1430236, 51479041, 51279038, 51309365).

\section{References}

[1] Molinari JF. Finite element simulation of shaped charges. Finite Elem Anal Des 2002;38(10):921-36.

[2] Hazell PJ, Lawrence T, Stennett C. The defeat of shaped charge jets by explosively driven ceramic and glass plates. Int J Appl Ceram Technol 2012;9 (2):382-92.

[3] Wu J, Liu J, Du Y. Experimental and numerical study on the flight and penetration properties of explosively-formed projectile. Int J Impact Eng 2007;34(7):1147-62.

[4] Sen S, Aksoy IG. An application of explosive metal forming in military field: the relationship between shaped charge jet formation and thickness variation along liner length of conical copper liner. Arabian J. Sci. Eng. 2013;38 (12):3551-62.

[5] Johnson GR, Stryk RA. Some considerations for 3D EFP computations. Int J Impact Eng 2006;32(10):1621-34.

[6] Katayama M, Kibe S, Yamamoto T. Numerical and experimental study on the shaped charge for space debris assessment. Acta Astronaut 2001;48 (5):363-72. 
[7] Feng DL, Liu MB, Li HQ, Liu GR. Smoothed particle hydrodynamics modeling of linear shaped charge with jet formation and penetration effects. Comput Fluids 2013;86:77-85.

[8] Liu MB, Liu GR, Zong Z, Lam KY. Computer simulation of high explosive explosion using smoothed particle hydrodynamics methodology. Comput Fluids 2003;32(3):305-22.

[9] Baêta-Neves AP, Ferreira A. Shaped charge simulation using SPH in cylindrical coordinates. Eng Comput 2015;32(2):370-86.

[10] Miyoshi H. Numerical simulation of shaped charges using the SPH solver: jet formation and target penetration. Mater Sci Forum 2008;566:65-70.

[11] Yang G, Han X, Hu DA. Computer simulation of two-dimensional linear-shaped charge jet using smoothed particle hydrodynamics. Eng Comput 2011;28 (1):58-75.

[12] Liu MB, Liu GR, Lam KY, Zong Z. Meshfree particle simulation of the detonation process for high explosives in shaped charge unlined cavity configurations. Shock Waves 2003;12(6):509-20.

[13] Niklas K, Kozak J. Experimental investigation of Steel-Concrete-Polymer composite barrier for the ship internal tank construction. Ocean Eng 2016:111:449-60.

[14] Huang CY, Chen YL. Design and impact resistant analysis of functionally graded $\mathrm{Al}_{2} \mathrm{O}_{3}-\mathrm{ZrO}_{2}$ ceramic composite. Mater Des 2016;91:294-305.

[15] Tasdemirci A, Tunusoglu G, Güden M. The effect of the interlayer on the ballistic performance of ceramic/composite armors: experimental and numerical study. Int J Impact Eng 2012;44:1-9.

[16] Zaera R, Sánchez-Sáez S, Pérez-Castellanos JL, Navarro C. Modelling of the adhesive layer in mixed ceramic/metal armours subjected to impact. Compos A Appl Sci Manuf 2000;31(8):823-33.

[17] Ali A, Sanuddin AB, Ezzeddin S. The effect of aging on Arenga pinnata fiberreinforced epoxy composite. Mater Des 2010;31(7):3550-4.

[18] Ma S, Mahfuz H. Finite element simulation of composite ship structures with fluid structure interaction. Ocean Eng 2012;52:52-9.

[19] Feng G, Li G, Liu Z, Niu HL, Li CF. Numerically simulating the sandwich plate system structures. J Mar Sci Appl 2010;9(3):286-91.

[20] Sharma S. Sandwich steels for crash energy absorption applications. University of Warwick; 2014.

[21] Kuo C, Sukovoy O. The Generic Management System (GMS) approach to fire safety of composite materials. Interfram 2004;2004.

[22] Kozak J. Selected problems on application of steel sandwich panels to marine structures. Polish Marit Res 2009:9-15.

[23] Phadnis VA, Makhdum F, Roy A, Silberschmidt VV. Drilling in carbon/epoxy composites: experimental investigations and finite element implementation. Compos A Appl Sci Manuf 2013;47:41-51.

[24] Isbilir O, Ghassemieh E. Numerical investigation of the effects of drill geometry on drilling induced delamination of carbon fiber reinforced composites. Compos Struct 2013;105:126-33.

[25] Shi Y, Swait T, Soutis C. Modelling damage evolution in composite laminates subjected to low velocity impact. Compos Struct 2012;94(9):2902-13.

[26] Børvik T, Hopperstad OS, Berstad T, Langseth M. Numerical simulation of plugging failure in ballistic penetration. Int J Solids Struct 2001;38 (34):6241-64.

[27] Ansari MM, Chakrabarti A. Impact behavior of FRP composite plate under low to hyper velocity impact. Compos B Eng 2016;95:462-74.

[28] Johnson GR, Stryk RA, Beissel SR. SPH for high velocity impact computations. Comput Methods Appl Mech Eng 1996;139(1):347-73.

[29] Johnson GR. Linking of Lagrangian particle methods to standard finite element methods for high velocity impact computations. Nucl Eng Des 1994;150(23):265-74

[30] Jianming W, Na G, Wenjun G. Abrasive waterjet machining simulation by SPH method. Int J Adv Manuf Technol 2010;50(1-4):227-34.

[31] Attaway SW, Heinstein MW, Swegle JW. Coupling of smooth particle hydrodynamics with the finite element method. Nucl Eng Des 1994;150 (2):199-205.

[32] Zhang ZC, Qiang HF, Gao WR. Coupling of smoothed particle hydrodynamics and finite element method for impact dynamics simulation. Eng Struct 2011;33(1):255-64.
[34] De Vuyst T, Vignjevic R, Campbell JC. Coupling between meshless and finite element methods. Int J Impact Eng 2005;31(8):1054-64.

[35] Fernández-Méndez S, Bonet J, Huerta A. Continuous blending of SPH with finite elements. Comput Struct 2005;83(17):1448-58.

[36] Yang Q, Jones V, McCue L. Free-surface flow interactions with deformable structures using an SPH-FEM model. Ocean Eng 2012:55:136-47.

[37] Chuzel-Marmot Y, Ortiz R, Combescure A. Three dimensional SPH-FEM gluing for simulation of fast impacts on concrete slabs. Comput Struct 2011;89 (23):2484-94.

[38] Liu X, Liu S, Ji H. Numerical research on rock breaking performance of water jet based on SPH. Powder Technol 2015;286:181-92.

[39] Zhang AM, Yang WS, Yao XL. Numerical simulation of underwater contact explosion. Appl Ocean Res 2012;34:10-20.

[40] Zhang AM, Yang WS, Chao H, Ming FR. Numerical simulation of column charge underwater explosion based on SPH and BEM combination. Comput Fluids 2013;71:169-78.

[41] Swegle JW, Attaway SW. On the feasibility of using smoothed particle hydrodynamics for underwater explosion calculations. Comput Mech 1995; 17:151-68.

[42] Colagrossi A, Landrini M. Numerical simulation of interfacial flows by smoothed particle hydrodynamics. J Comput Phys 2003;191:448-75.

[43] Zhang ZF, Ming FR, Zhang AM. Damage characteristics of coated cylindrical shells subjected to underwater contact explosion. Shock Vib 2014.

[44] Zhang ZF, Sun LQ Yao XL, Cao XY. Smoothed particle hydrodynamics simulation of the submarine structure subjected to a contact underwater explosion. Combust Explosion, Shock Waves 2015;51(4):502-10.

[45] Zhang AM, Sun PN, Ming FR. An SPH modeling of bubble rising and coalescing in three dimensions. Comput Methods Appl Mech Eng 2015;294:189-209.

[46] Cao XY, Ming FR, Zhang AM. Sloshing in a rectangular tank based on SPH simulation. Appl Ocean Res 2014;47:241-54.

[47] Ming FR, Zhang AM, Xue YZ, Wang SP. Damage characteristics of ship structures subjected to shockwaves of underwater contact explosions. Ocean Eng 2016;117:359-82.

[48] Drathi R, Das AJM, Rangarajan A. Meshfree simulation of concrete structures and impact loading. Int J Impact Eng 2016;91:194-9.

[49] Liu MB, Liu GR. Smoothed particle hydrodynamics (SPH): an overview and recent developments. Arch Comput Methods Eng 2010;17:25-76.

[50] Zhang AM, Ming FR, Cao XY. Total Lagrangian particle method for the largedeformation analyses of solids and curved shells. Acta Mech 2014;225 (1):253-75.

[51] Liu GR, Liu MB. Smoothed particle hydrodynamics - A meshfree particle method. Singapore: World Scientific Publishing Co., Pte. Ltd; 2003.

[52] Dobratz BM, LLNL Explosive Handbook: properties of chemical explosives and explosives and explosive simulants. Report UCRL-52997, Lawrence Livermore National Laboratory, Livermore, CA (USA); 1981.

[53] Allahdadi FA, Carney TC, Hipp JR, Libersky LD, Petschek AG. High strain lagrangian hydrodynamics a three dimensional SPH code for dynamic material response. J Comput Phys 1993;109:67-75.

[54] Johnson GR, Cook WH. A constitutive model and data for metals subjected to large strains, high strain rates and high temperatures. Proc. 7th International Symposium on Ballistics, USA, vol. 21. p. 541-7.

[55] Hallquist JO, LS-DYNA Theory Manual. Livermore Software Technology Corporation; 2006.

[56] Cowper G, Symonds P. Strain-hardening and strain-rate effects in the impact loading of cantilever beams. Technical report, Brown University, Division of Applied Mathematics; 1957.

[57] Yan F, Li YZ. Analysis on crashworthiness of FPSO broadside based on Sandwich Plate System (SPS). Guangdong Shipbuild 2014:6:70-2 (in Chinese).

[58] Campbell J, Vignjevic R, Libersky L. A contact algorithm for smoothed particle hydrodynamics. Comput Methods Appl Mech Eng 2000;184(1):49-65.

[59] Hiermaier S, Könke D, Stilp AJ, Thoma K. Computational simulation of the hypervelocity impact of Al-spheres on thin plates of different materials. Int J Impact Eng 1997;20(1):363-74. 